\title{
A quadratic bound on the number of boundary slopes of essential surfaces with bounded genus
}

\author{
Tao $\mathrm{Li}^{*} \quad$ Ruifeng Qiu ${ }^{\dagger} \quad$ Shicheng Wang ${ }^{\ddagger}$
}

\begin{abstract}
Let $M$ be an orientable 3-manifold with $\partial M$ a single torus. We show that the number of boundary slopes of immersed essential surfaces with genus at most $g$ is bounded by a quadratic function of $g$. In the hyperbolic case, this was proved earlier by Hass, Rubinstein and Wang.
\end{abstract}

Subject class: 57M50, 57N10

\section{Introduction}

A proper immersion $f:(F, \partial F) \rightarrow(M, \partial M)$ from a compact surface to a compact 3 -manifold is essential if it is $\pi_{1}$-injective and $\partial$-injective, i.e., it maps essential loops and $\operatorname{arcs}$ in $F$ to essential loops and $\operatorname{arcs}$ in $M$. Let $M$ be a compact orientable 3 -manifold with $\partial M$ a single torus. We say a slope $s$ in $\partial M$ is realized by an essential surface if there is a proper essential immersion $f:(F, \partial F) \rightarrow(M, \partial M)$ such that every component of $f(\partial F)$ is a curve of slope $s$ in $\partial M$. Such an immersed surface is particularly interesting because it extends to a closed immersed surface in the closed 3-manifold $M(s)$ obtained by Dehn filling along the slope $s$.

The study of boundary slopes of essential surfaces has been an active and attractive topic for long times. Hatcher [Ha] showed that there are only finitely many boundary slopes of embedded essential surfaces. The number of boundary slopes of small-genus embedded surfaces (e.g. punctured spheres or tori) is quite small and the study of these exceptional slopes is a center topic in the theory of Dehn surgery, see the survey article [Go].

${ }^{*}$ Partially supported by NSF grant DMS-0705285

${ }^{\dagger}$ Partially supported by NSFC grant 10631060

†Partially supported by NSFC grant 10625102 
However, for immersed essential surfaces, there is no such bound in general. In fact, there are examples that every slope is realized by an immersed essential surface, see [Ba, $[\mathrm{BC}, \mathrm{O}]$. In [HRW], Hass, Rubinstein and Wang show that for hyperbolic manifolds, the number of boundary slopes of essential surfaces of genus at most $g$ is bounded by $C g^{2}$, where $C$ is a constant independent of the manifold (see also Agol $[\mathrm{Ag}]$ ). The purpose of this paper is to extend the quadratic bound result to general 3-manifolds.

Theorem 1.1. Suppose $M$ is an orientable 3-manifold with $\partial M$ a single torus. For any $g$, let $N_{g}(M)$ be the number of slopes that can be realized by essential immersed surfaces of genus at most $g$.

\section{depend on $M$.}

Then $N_{g}(M) \leq\left\{\begin{array}{cc}C(M) g^{2} & g \geq 1 \\ C^{\prime}(M) & g=0\end{array}\right.$ for some constants $C(M)$ and $C^{\prime}(M)$ that

Remark. (1). In [HRW], Hass, Rubinstein and Wang proved that $N_{g}(M)$ is finite, but no bound on $N_{g}(M)$ is given in [HRW]. Recently Zhang [Zh extended the techniques in [HRW] and proved that $N_{g}(M)$ is bounded by $c(M) g^{3}$ for some constant $c(M)$ that depends on $M$.

(2). The coefficient $C(M)$ depends on $M$. One would hope for a quadratic bound independent of $M$, but even for embedded surfaces, it seems difficult to obtain such a bound if $M$ contains essential annuli. Nevertheless, the coefficients $C(M)$ and $C^{\prime}(M)$ can be algorithmically determined, see Remark 4.4,

(3). When $\partial M$ is a high genu surface, there are finiteness and infiniteness results in both embedded and immersed case, see [SWu, Qi], [HWZ], [La] and QW].

\section{Some crucial facts}

The proof of Theorem 1.1 relies on a theorem of Hass-Rubinstein-Wang [HRW], a theorem of Culler-Shalen [CS], and Li's extension of Hatcher's argument [Li2]. Propositions 2.1 , 2.2 and 2.3 below are their variations, presented in the forms we need.

In this section we first consider a hyperbolic 3-manifold $M$ with possibly more than one cusp. We denote by $M_{\max }$ the interior of $M$ with a system of maximal cusps removed. Now we identify $M$ with $M_{\max }$, then $\partial M$ has a Euclidean metric induced from the hyperbolic metric and each closed Euclidean geodesic in $\partial M$ has length at least 1 (see [Ad] for detail).

Proposition 2.1. Suppose $M$ is a hyperbolic 3-manifold as above and $T$ is a component of $\partial M$. Suppose $F$ is an essential immersed surface of genus $g$ in $M$ and let $c_{1}, \ldots, c_{n}$ be the components of $\partial F \cap T$. 
1. If we identify $M$ with $M_{\max }$, then

$$
\sum_{i=1}^{n} L\left(c_{i}\right) \leq-2 \pi \chi(F),
$$

where $L\left(c_{i}\right)$ is the length of an Euclidean geodesic homotopic to $c_{i}$ in $T$.

2. Let $S$ be an embedded essential surface in $M$ and let $\gamma$ be a component of $\partial S \cap T$. Then there is a number $C_{S}$ which can be expressed as an explicit function of $\chi(S)$, such that

$$
|\gamma \cap \partial F| \leq-C_{S} \cdot \chi(F),
$$

where $|\gamma \cap \partial F|$ is the minimum number of intersection points of $\gamma$ and $\partial F$.

3. There are two distinct essential circles $\Gamma_{1}$ and $\Gamma_{2}$ in $T$, such that

$$
\left|\Gamma_{j} \cap \partial F\right| \leq-C \chi(F)
$$

for some constant $C$, where $\left|\Gamma_{j} \cap \partial F\right|$ is the minimum number of intersection points of $\Gamma_{j}$ and $\partial F$ up to isotopy, $j=1,2$.

Proof. Part (1) is proved in [HRW].

Now we prove part (2). Recall we have identified $M$ with $M_{\max }$, and $\partial M$ has a Euclidean metric induced from the hyperbolic metric. We may assume that $\gamma$ and each component $c_{i}$ of $\partial F$ have been isotoped to be closed Euclidean geodesics in $\partial M$.

Let $p: E^{2} \rightarrow T$ be the universal cover, where $E^{2}$ is the Euclidean plane. By lifting $\gamma$ to $E^{2}$, we get an Euclidean line segment $O O_{1}$ which projects to $\gamma$. By part (1), the Euclidean length $L(\gamma)=L\left(O O_{1}\right)$ is at most $-2 \pi \chi(S)$. The covering translations of $O$ form a lattice in $E^{2}$. Let $O_{2}$ be a lattice point such that $O O_{1}$ and $O O_{2}$ span a fundamental parallelogram $P$ for $T$. By a theorem of Cao and Meyerhoff (also see Lemma 2.2 of [HRW], area $(P) \geq 3.35$.

Let $h$ be the distance from $O_{2}$ to the line $O O_{1}$. Since the Euclidean length $L\left(O O_{1}\right) \leq-2 \pi \chi(S)$ and since area $(P) \geq 3.35$, the height $h \geq \frac{3.35}{L\left(O O_{1}\right)} \geq \frac{3.35}{-2 \pi \chi(S)}$.

By lifting $c_{i}$ to $E^{2}$, it is easy to see that the length of $c_{i}$ is at least $h\left|c_{i} \cap \gamma\right|$. By part (1), we have

$$
h|\gamma \cap \partial F|=h \sum_{i=1}^{n}\left|c_{i} \cap \gamma\right| \leq \Sigma_{i=1}^{n} L\left(c_{i}\right) \leq-2 \pi \chi(F) .
$$

So part (2) holds and $C_{S}=\frac{-4 \pi^{2} \chi(S)}{3.35}$.

The proof of part (3) is similar. Pick an origin $O$ in $E^{2}$ and consider the lattice $L$ in $E^{2}$ given by the covering translations of $O$. Let $O_{1}$ and $O_{2}$ be two independent vertices in $L$ which have the first and second shortest distance from the origin $O$. Let $\alpha$ be the angle of the triangle $O O_{1} O_{2}$ at $O$ and let $l, l_{1}, l_{2}$ be the lengths of $O_{1} O_{2}$, 
$O O_{1}$ and $O O_{2}$ respectively. By our assumptions above and by a theorem in $\mathrm{Ad}$ ] mentioned earlier, we have $l \geq l_{2} \geq l_{1} \geq 1$. This implies that $\alpha \geq \pi / 3$. Furthermore, we can assume that $\alpha \leq \pi / 2$, because otherwise we can replace one of the vertices by its inverse.

$O O_{1}$ and $O O_{2}$ span a fundamental parallelogram $P$ for $T$. It follows from our assumptions above that $l_{1} \sin \alpha$ (resp. $l_{2} \sin \alpha$ ), the height of $P$ over $O O_{2}$ (resp. over $\left.O O_{1}\right)$, is at least $\frac{\sqrt{3}}{2}$. Let $\Gamma_{j}=p\left(O O_{j}\right), j=1,2$. As in part (2), we have

$$
\frac{\sqrt{3}}{2}\left|\Gamma_{j} \cap \partial F\right|=\frac{\sqrt{3}}{2} \sum_{i=1}^{n}\left|c_{i} \cap \Gamma_{j}\right| \leq \sum_{i=1}^{n} L\left(c_{i}\right) \leq-2 \pi \chi(F),
$$

and part (3) follows with $C=\frac{4 \pi}{\sqrt{3}}$.

Proposition 2.2. Suppose $M$ is a hyperbolic 3-manifold as above and $T$ is a component of $\partial M$. Then $T$ has two distinct boundary slopes $c_{1}$ and $c_{2}$ of embedded essential surfaces, i.e., there are properly embedded essential surfaces $F_{i}$ in $M$ such that $F_{i} \cap T$ is a multiple of $c_{i}, i=1,2$.

Proof. By performing hyperbolic Dehn filling on each boundary component of $\partial M \backslash T$, we get a hyperbolic 3-manifold $M^{*}$ with $\partial M^{*}=T$. By a theorem of Culler-Shalen [CS], there are two distinct boundary slopes $c_{1}$ and $c_{2}$ on $T$, i.e. there are properly embedded essential surfaces $F_{i}^{*}$ in $M^{*}$ such that $F_{i}^{*} \cap T$ is a multiple of $c_{i}, i=1,2$. So $F_{i}=F_{i}^{*} \cap M$ has the required property.

A surface in a Seifert fiber space is said to be horizontal if it is transverse to the $S^{1}$-fibers. If an orientable Seifert fiber space has a single boundary component, then it is easy to see that all embedded horizontal surfaces have the same slope which is determined by its Euler number. The following Lemma is a generalization of this fact to immersed horizontal surfaces in a Seifert fiber space with more than one boundary component.

Proposition 2.3. Let $N$ be an orientable Seifert fiber space with boundary and $T$ a boundary component of $N$. Let $F_{1}$ and $F_{2}$ be immersed essential horizontal surfaces in $N$. Suppose $F_{i} \cap T$ is embedded for both $i=1,2$ and $\left|\partial F_{1} \cap \partial F_{2} \cap T\right|$ is minimal in the isotopy classes of $F_{1}$ and $F_{2}$. If there is a double curve $\alpha \subset F_{1} \cap F_{2}$ with both endpoints in $T$, then the curves of $F_{1} \cap T$ and $F_{2} \cap T$ must have the same slope in $T$.

Proof. The proof of the lemma is basically an argument first used by Hatcher in Ha and then extended to immersed surfaces in [Li2]. As $N$ is a Seifert fiber space, we can fix a direction for the $S^{1}$-fibers of $N$ in $T$. Since $N$ is orientable and each $F_{i}$ is horizontal, the normal direction of $\partial N$ and the orientation of the $S^{1}$-fibers in $T$ uniquely determine an orientation for every curve of $\partial F_{1} \cap T$ and $\partial F_{2} \cap T$. Since 
$F_{i} \cap T$ is embedded, every component of $\partial F_{i} \cap T(i=1$ or 2$)$ with this induced orientation represents the same element in $H_{1}(T)$. If $\partial F_{1} \cap T$ and $\partial F_{2} \cap T$ have different slopes, they must have a nonzero intersection number. Moreover, since we have assumed $\left|\partial F_{1} \cap \partial F_{2} \cap T\right|$ is minimal in the isotopy classes of $F_{1}$ and $F_{2}$, the signs of the intersection points of $\partial F_{1} \cap \partial F_{2} \cap T$ (with respect to the directions above) are the same, either all positive or all negative.

Let $\alpha \subset F_{1} \cap F_{2}$ be an intersection arc with both endpoints in $T$. One can easily list all possible configurations of the directions of the $S^{1}$-fibers at $\partial \alpha$ and the induced orientations of $\partial F_{1}$ and $\partial F_{2}$. However, since each $F_{i}$ is horizontal, only two possible configurations can happen, see Figure 1, In either case, the two ends of $\alpha$ give points of $\partial F_{1} \cap \partial F_{2} \cap T$ with opposite signs of intersection. This contradicts our conclusion on the sign of the intersection points above. So $F_{1} \cap T$ and $F_{2} \cap T$ must have the same slope in $T$.
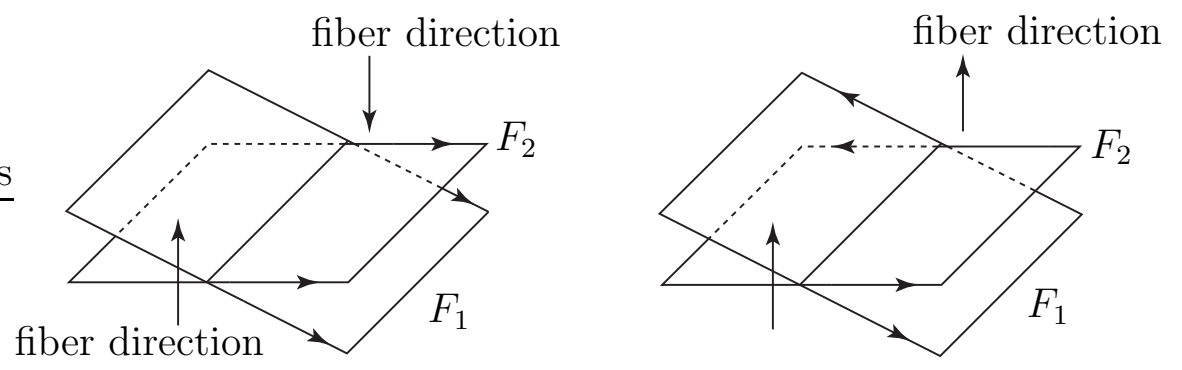

Figure 1:

The following fact follows immediately from Lemma 2.3. Since an essential surface in a Seifert fiber space is either vertical or horizontal [H], if $M$ is an orientable Seifert fiber space with a single boundary component, this means that only two possible slopes can be realized by immersed essential surfaces, one vertical and one horizontal.

Corollary 2.4. Let $N$ be an orientable Seifert fiber space with a single boundary torus. Then all immersed horizontal surfaces with respect to a fixed Seifert structure have the same slope in $\partial N$.

\section{Construct a surface of reference}

Let $M$ be as in Theorem 1.1. First note that we may assume $M$ is irreducible, since if $M$ is reducible we can use the prime factor of $M$ that contains $\partial M$ and the proof 
is the same. Since the hyperbolic case is proved in [HRW] and the Seifert fiber case is trivial (see Corollary 2.4), we may assume $M$ has a nontrivial JSJ decomposition.

Let $\mathcal{T}$ be the set of JSJ decomposition tori of $M$. We call the closure (under path metric) of each component of $M-N(\mathcal{T})$ a JSJ piece. Let $M_{0}$ be the JSJ piece that contains the torus $\partial M$.

In this section, we suppose $M_{0}$ is a Seifert fiber space and we will use the JSJ structure of $M$ to construct a surface of reference for counting the boundary slopes of immersed essential surfaces. This surface is in $M_{0}$ and is not a proper surface in $M$.

For any Seifert fiber space $N$ with boundary, we call a slope in a boundary torus the vertical slope if it is the slope of a regular fiber of $N$.

Proposition 3.1. Let $N$ be a Seifert fiber space and $T_{0}, T_{1}, \ldots T_{n}$ the boundary tori of $N$. Let $s_{i}(i=1, \ldots n)$ be any slope in $T_{i}$ that is not vertical in $N$. Then there is an embedded horizontal surface in $N$ realizing each slope $s_{i}$ in $T_{i}$.

Proof. We perform Dehn fillings along each slope $s_{i}(i=1, \ldots n)$ and let $\hat{N}$ be the resulting manifold. So $\partial \hat{N}=T_{0}$. Since $s_{i}$ is not vertical in $N$, the Seifert structure of $N$ extends to $\hat{N}$. Hence $\hat{N}$ is a Seifert fiber space with boundary. Every Seifert fiber space with boundary has an embedded horizontal surface. The restriction of a horizontal surface of $\hat{N}$ to $N$ is a horizontal surface of $N$ realizing each slope $s_{i}$ in $T_{i}$ $(i=1, \ldots n)$.

Let $M_{0}$ be the Seifert JSJ piece of $M$ as above. Let $\partial M, T_{1}, \ldots, T_{n}$ be the boundary tori of $M_{0}$. So each $T_{i}$ can be viewed as a JSJ torus in $\mathcal{T}$ and $M_{0}$ is a JSJ piece on one side of $T_{i}$. Next we fix a slope in $T_{i}$ according the JSJ piece on the other side of $T_{i}$. Let $M_{i}$ be the JSJ piece on the other side of $T_{i}$. Note that $M_{i}$ is the same as $M_{0}$ if $T_{i}$ is glued to some $T_{j}$ in $M$. We fix a slope $s_{i}$ for each boundary component $T_{i}$ of $M_{0}$ as follows.

Case 1. $M_{i}$ is a Seifert fiber space and $M_{i}$ is not a twisted $I$-bundle of a Klein bottle. In this case we choose the slope $s_{i}$ of $T_{i}$ to be the slope of a regular fiber of $M_{i}$. Note that $s_{i}$ is not a vertical slope for $M_{0}$, because otherwise the regular fibers of $M_{0}$ and $M_{i}$ match and $M_{0} \cup_{T_{i}} M_{i}$ is a Seifert fiber space, which contradicts the hypothesis that $T_{i}$ is a JSJ torus. So $s_{i}$ is not a vertical slope for $M_{0}$.

Case 2. $M_{i}$ is a twisted $I$-bundle over a Klein bottle. In this case, $M_{i}$ has two different Seifert structures [Ja]. For any point $x \in T_{i}=\partial M_{i}$, we define $p(x)$ to be the other endpoint of the $I$-fiber of $M_{i}$ that contains $x$. Let $\gamma_{\nu}$ be a simple closed curve in $T_{i}$ which is a regular fiber of $M_{0}$. Let $s_{i}$ be the slope of $p\left(\gamma_{\nu}\right)$. Note that $\gamma_{\nu}$ and $p\left(\gamma_{\nu}\right)$ 
bound an immersed essential annulus in $M_{i}$. If $\gamma_{\nu}$ and $p\left(\gamma_{\nu}\right)$ have the same slope in $T_{i}$, i.e. $\gamma_{\nu} \cup p\left(\gamma_{\nu}\right)$ bounds an embedded annulus, then we can choose a Seifert structure for $M_{i}$ [Ja] so that $\gamma_{\nu}$ is also a regular fiber for $M_{i}$ and hence $M_{0} \cup M_{i}$ is a Seifert fiber space, a contradiction to the hypothesis that $T_{i}$ is a JSJ torus. So $s_{i}$ is not a vertical slope for $M_{0}$.

Case 3. $M_{i}$ is hyperbolic. By Proposition 2.2, $T_{i}$ has at least two boundary slopes (of embedded essential surfaces in $M_{i}$ ). In this case we choose $s_{i}$ to be a boundary slope of $M_{i}$ that is not a vertical slope in $M_{0}$. So there is an embedded essential surface $S_{i}$ in $M_{i}$ whose boundary in $T_{i}$ has slope $s_{i}$ and $s_{i}$ is not a vertical slope in $M_{0}$.

By Proposition 3.1, $M_{0}$ contains a properly embedded horizontal surface $S$ such that the slope of $\partial S \cap T_{i}$ is the slope $s_{i}$ described above. Note that $S$ is not a properly embedded surface in $M$, since two tori $T_{i}$ and $T_{j}(i \neq j)$ may be glued together in $M$ and $s_{i}$ and $s_{j}$ may not match in the corresponding JSJ torus of $M$.

Next we fix the surface $S$ in the construction above. Let $\mu$ the slope of $S \cap \partial M$ in the torus $\partial M$ and let $\nu$ be the vertical slope of $\partial M$ with respect to the Seifert structure of $M_{0}$.

\section{Proof of the Theorem 1.1}

Let $F$ be a proper immersed essential surface of genus $g$ in $M$.

If $M_{0}$ is hyperbolic then Theorem 1.1 follows from [HRW]. More precisely, suppose $\partial F$ is an $n$ multiple of a slope $c$ in $\partial M$ and we have identified $M_{0}$ with the metric space $M_{0 \max }$ as in Proposition 2.1, By Proposition 2.1 (1), we have

$$
n L(c) \leq L\left(\partial\left(F \cap M_{0}\right)\right) \leq-2 \pi \chi\left(F \cap M_{0}\right) \leq-2 \pi \chi(F)=2 \pi(2 g-2+n) .
$$

Then as discussed in HRW we have $L(c) \leq 2 \pi$ if $g=0$ and $L(c) \leq 2 g \pi$ if $g>0$, therefore $N_{g}(M) \leq C^{\prime}$ for $g=0$ and $N_{g}(M) \leq C g^{2}$ for some constants $C^{\prime}$ and $C$ independent of $M$.

Below we assume that $M_{0}$ is a Seifert fiber space.

We may assume the slope of $\partial F$ is not the vertical slope of $M_{0}$, so $F \cap M_{0}$ is horizontal in $M_{0}$. Since $\partial M$ is incompressible, $F$ is not a disk. If $F$ is an annulus, then $F \cap M_{0}$ is a horizontal annulus. The only orientable Seifert fiber space that admits a horizontal annulus is either $T^{2} \times I$ or a twisted $I$-bundle over a Klein bottle. Since $M_{0}$ is a JSJ piece, $M_{0}$ is not $T^{2} \times I$. If $M_{0}$ is a twisted $I$-bundle over a Klein bottle, $M_{0}=M$ and by Corollary 2.4 there are only two possible slopes for $F$. Thus Theorem 1.1 holds if $\chi(F) \geq 0$. So in this section, we assume $\chi(F)<0$. 
Lemma 4.1. Let $N$ be a Seifert JSJ piece of $M$ and $v$ a regular fiber of $N$. Suppose $N$ is not a twisted I-bundle over a Klein bottle. Let $F$ be an essential surface in $M$ and suppose $F \cap N$ is horizontal in $N$. Then $|v \cap F| \leq-6 \chi(F)$.

Proof. Let $O(N)$ be the base orbifold of $N$. Since $O(N)$ has boundary and $N$ is not a solid torus, $\chi(O(N)) \leq 0$. Moreover, since $N$ is orientable and is not $T^{2} \times I$, $\chi(O(N))=0$ if and only if $O(N)$ is a disk with two cone points both of order 2 and $N$ is a twisted $I$-bundle over a Klein bottle. Thus by our hypothesis that $N$ is not a twisted $I$-bundle over a Klein bottle, we have $\chi(O(N))<0$.

Since $F \cap N$ is horizontal in $N, \chi(F \cap N)=k \chi(O(N))$ where $k=|v \cap F|$. Since $O(N)$ has boundary, the maximal possible value for $\chi(O(N))$ occurs when $O(N)$ is a disk with two cone points of orders 2 and 3 respectively, in which case $\chi(O(N))=$ $-1 / 6$. Therefore $\chi(O(N)) \leq-1 / 6$ and $k=|v \cap F| \leq-6 \chi(F \cap N) \leq-6 \chi(F)$.

Remark 4.2. In the proof of Lemma 4.1, $\chi(O(N)) \leq-1 / 2$ except when $O(N)$ is a disk with two cone points. Thus $|v \cap F| \leq-2 \chi(F)$ if $\partial N$ has more than one boundary component. This is a key observation in the proof of the following lemma, see [Zh].

Lemma 4.3 ([Zh], Lemma 3.2). Let $M$ and $M_{0}$ be as in section 3 and let $\nu$ be the vertical slope of $\partial M$ in $M_{0}$. Let $F$ be an immersed essential surface in $M$ of genus at most $g$ and let $s_{F}$ be the boundary slope of $F$ in $\partial M$. Then the geometric intersection number

$$
\Delta\left(\nu, s_{F}\right) \leq U(g)=\left\{\begin{array}{cc}
2 & g=0 \\
2 g & g \geq 1
\end{array}\right. \text {. }
$$

Proof of Theorem 1.1 when $M_{0}$ is a Seifert fiber space. Let $S$ be the fixed embedded horizontal surface in $M_{0}$ constructed in section 3 , Let $F$ be an immersed essential surface in $M$ of genus at most $g$. We will study the intersection of $F \cap M_{0}$ and $S$. Let $s_{F}$ be the boundary slope of $F$. Our main goal is to show that $\Delta\left(\mu, s_{F}\right)$ is bounded by a linear function of $g$, where $\mu$ is the slope of $\partial S \cap \partial M$. As only one slope is vertical, we suppose $F \cap M_{0}$ is horizontal in $M_{0}$.

We will use the same notation as section 3. The boundary tori of $M_{0}$ are $\partial M$, $T_{1}, \ldots, T_{n}$ and $S$ is properly embedded in $M_{0}$. In this section, we view $S$ as a surface in $M$ instead of $M_{0}$. Since it is possible that $T_{i}$ and $T_{j}(i \neq j)$ are glued together in $M$, when regarded as a surface in $M$, curves of $\partial S$ may intersect in a JSJ torus of $M$.

Now we consider the intersection of $F$ and $S$. A key difference between $F$ and $S$ is that $F$ is a proper surface in $M$ while $S$ is only defined in $M_{0}$. We view the torus $T_{i}$ as a JSJ torus of $M$ and as in section 3 , let $M_{i}$ be the JSJ piece incident to $T_{i}$ on the other side of $M_{0}\left(M_{i}\right.$ may be the same JSJ piece as $\left.M_{0}\right)$. Let $\Gamma_{i}=S \cap T_{i}$ in $M_{0}$. 
As above, we view $\Gamma_{i}$ as a collection of curves in a JSJ torus in $M$. Next we estimate $\left|F \cap \Gamma_{i}\right|$. Let $k_{i}$ be the number of components of $\Gamma_{i}$. As in the construction of $S$, we have 3 cases:

Case 1. $M_{i}$ is a Seifert fiber space and $M_{i}$ is not a twisted $I$-bundle over a Klein bottle. By the construction of $S$, in this case, each curve in $\Gamma_{i}$ is a regular fiber of the Seifert fiber space $M_{i}$. By Lemma 4.1, $\left|F \cap \Gamma_{i}\right| \leq-6 k_{i} \chi\left(F \cap M_{i}\right) \leq-6 k_{i} \chi(F)$.

Case 2. $M_{i}$ is a twisted $I$-bundle over a Klein bottle. By our construction of $S$ in this case, each curve $\gamma$ in $\Gamma_{i}$ and a regular fiber $p(\gamma)$ of $M_{0}$ bound an immersed essential annulus in $M_{i}$. We may assume $F \cap M_{i}$ to be essential in $M_{i}$. So the intersection of $F$ and an essential annulus in $M_{i}$ consists of essential arcs in the annulus. In particular, $|F \cap \gamma|=|F \cap p(\gamma)|$. Since $p(\gamma)$ is a regular fiber of $M_{0}$ for each curve $\gamma$ in $\Gamma_{i}$ and since $M_{0}$ has more than one boundary component, by Lemma 4.1 and Remark 4.2, $\left|F \cap \Gamma_{i}\right| \leq-2 k_{i} \chi\left(F \cap M_{0}\right) \leq-2 k_{i} \chi(F)$.

Case 3. $M_{i}$ is hyperbolic. In this case there is an embedded essential surface $S_{i}$ in $M_{i}$ whose boundary slope in the torus $T_{i}$ is the same as the slope of $\Gamma_{i}$. Now we consider the intersection of $S_{i}$ and $F \cap M_{i}$. By Proposition $2.1(2),\left|F \cap \Gamma_{i}\right| \leq-c_{i} \chi\left(F \cap M_{i}\right) \leq$ $-c_{i} \chi(F)$ for some number $c_{i}$ which depends on $\left|\Gamma_{i}\right|$ and $\chi\left(S_{i}\right)$.

Let $\Gamma_{0}=\partial S \cap \partial M$ be the boundary curves of $S$ lying in $\partial M$. So $\partial S-\Gamma_{0}=\bigcup_{i=1}^{n} \Gamma_{i}$. By the argument above, there is a number $c>0$ depending on $S$ such that the total number of intersection points of $F$ and $\partial S-\Gamma_{0}$ is at most $-c \chi(F)=c(2 g-2+|\partial F|)$ for some constant $c$ which depends on $\left|\partial S-\Gamma_{0}\right|$ and the surface $S_{i}$ in the case that $M_{i}$ is hyperbolic as in Case (3).

Let $\Delta=\Delta\left(\mu, s_{F}\right)$ be the intersection number of a curve in $\Gamma_{0}$ and a curve $\partial F$. So the total number of intersection points of $\Gamma_{0}$ and $\partial F$ is $\Delta \cdot\left|\Gamma_{0}\right| \cdot|\partial F|$.

Thus, if $g \geq 1$, there is a number $C_{1}$ depending on $S$ and $S_{i}$ such that if $\Delta>C_{1} g$, we have $\Delta \cdot\left|\Gamma_{0}\right| \cdot|\partial F|>c(2 g-2+|\partial F|)$ and hence there must be an arc in $S \cap F$ with both endpoints in $\partial M$. However, by Proposition 2.3 , this means that $\partial F$ has the same slope as $\partial S \cap \partial M$ and $\Delta=0$, a contradiction. Therefore, if $g \geq 1, \Delta \leq C_{1} g$ for some constant $C_{1}$ which depends on $S$ and the surface $S_{i}$ in Case (3).

Similarly if $g=0$, there is a number $C_{0}$ such that if $\Delta>C_{0}$, then $\Delta \cdot\left|\Gamma_{0}\right| \cdot|\partial F|>$ $c(|\partial F|-2)$ and hence there must be an arc in $S \cap F$ with both endpoints in $\partial M$, which means that $\Delta=0$. Thus if $g=0, \Delta \leq C_{0}$ for some constant $C_{0}$ which depends on $M$.

We have two fixed slopes for $\partial M$, the vertical slope $\nu$ and the slope $\mu$ of $\partial S \cap \partial M$. For any horizontal immersed essential surface $F$ of genus at most $g$, let $s_{F}$ be its 
boundary slope. The argument above says that $\Delta\left(\mu, s_{F}\right) \leq V(g)$, where $V(g)=C_{1} g$ if $g \geq 1$ and $V(g)=C_{0}$ is $g=0$ for some constants $C_{1}$ and $C_{0}$ depending on $S$. By Lemma 4.3, $\Delta\left(\nu, s_{F}\right) \leq U(g)$ where $U(g)=2 g$ if $g \geq 1$ and $U(g)=2$ if $g=0$. Therefore, the total number of possible slopes for $\partial F$ is bounded by a quadratic function of $g$, where the coefficients depend on the fixed surface $S$ and the surface $S_{i}$ used in the hyperbolic JSJ piece as in Case (3).

Remark 4.4. If one uses part (3) of Proposition 2.1 instead of part (2) in the argument, then one can prove the main theorem without using the Culler-Shalen theorem (i.e. Proposition 2.2). However, there is an advantage of using Proposition 2.2. Given any triangulation of a 3-manifold, one can use normal surface theory to algorithmically find two embedded essential surfaces with different boundary slopes whose existence is guaranteed by Proposition 2.2. Since there are algorithms to determine the JSJ and Seifert structures, the constant in Theorem 1.1 can be found algorithmically by following the proof.

\section{References}

[Ad] Colins Adams, Volumes of $N$-cusped hyperbolic 3-manifolds, J. London Math. Soc. 38 (1988) 555-565.

[Ag] Ian Agol, Bounds on exceptional Dehn filling. Geom. Topol. 4 (2000), 431449

[Ba] Mark Baker, On boundary slopes of immersed incompressible surfaces. Ann. Inst. Fourier (Grenoble) 46 (1996), no. 5, 1443-1449.

[BC] Mark Baker, Daryl Cooper, A combination theorem for convex hyperbolic manifolds, with applications to surfaces in 3-manifolds. J. Topol. 1 (2008), no. 3, 603-642.

[CS] Marc Culler, Peter Shalen, Bounded, separating, incompressible surfaces in knot manifolds. Inven. Math. 75. (1984) 537-545.

[Go] Cameron McA Gordon, Small surfaces and Dehn filling. Proceedings of the Kirbyfest (Berkeley, CA, 1998), 177-199 , Geom. Topol. Monogr., 2, 1999.

[H] Joel Hass, Minimal surfaces in manifolds with $S^{1}$ actions and the simple loop conjecture for Seifert fiber spaces. Proc. Amer. Math. Soc. 99 (1987) 383-388.

[HRW] Joel Hass, Hyam Rubinstein, Shicheng Wang, Boundary slopes of immersed surfaces in 3-manifolds. J. Differential Geometry 52 (1999) 303-325. 
[HWZ] Joel Hass, Shicheng Wang, Qing Zhou, On finiteness of the number of boundary slopes of immersed surfaces in 3-manifolds. Proc. Amer. Math. Soc. 130 (2002), no. 6, 1851-1857

[Ha] Allen Hatcher, On the boundary curves of incompressible surfaces. Pacific J. Math. 99 (1982), 373-377.

[Ja] William Jaco, Lectures on Three-Manifold Topology. CBMS Regional Conference Series in Mathematics, 43 (1977).

[La] Lackenby, Marc Attaching handlebodies to 3-manifolds. Geom. Topol. 6 (2002), 889-904

[Li1] Tao Li, Immersed essential surfaces in hyperbolic 3-manifolds. Comm. Anal. Geom. 10 (2002), 275-290.

[Li2] Tao Li, Boundary curves of surfaces with the 4-plane property. Geometry \& Topology, 6 (2002), 609-647.

[O] Ulrich Oertel, Boundaries of $\pi_{1}$-injective surfaces. Topology and its Applications, 78 (1997) 215-234.

[Qi] Ruifeng Qiu, Incompressible surfaces in handlebodies and closed 3-manifolds of Heegaard genus 2, Proc. Amer. Math. Soc. 128:10 (2000), 3091-3097.

[QW] Ruifeng Qiu, Shicheng Wang, Handle additions producing essential surfaces. Pacific J. Math. 229 (2007), no. 1, 233-255.

[SWu] Martin Scharlemann, Ying-Qing Wu, Hyperbolic manifolds and degenerating handle additions, J. Austral. Math. Soc. Ser. A 55:1 (1993), 72-89.

[Zh] Qiang Zhang, Boundary slopes of immersed surfaces in Haken manifolds. Journal of Knot Theory and its ramification, to appear.

Department of Mathematics; Boston College; Chestnut Hill, MA 02167 USA.

Email address: taoli@bc.edu

Department of Mathematics; East China Normal University; Shanghai 200062 CHINA

Email address: qiurf@dlut.edu.cn

Department of Mathematics; Peking University, Beijing 100871, CHINA

Email address: wangsc@math.pku.edu.cn 